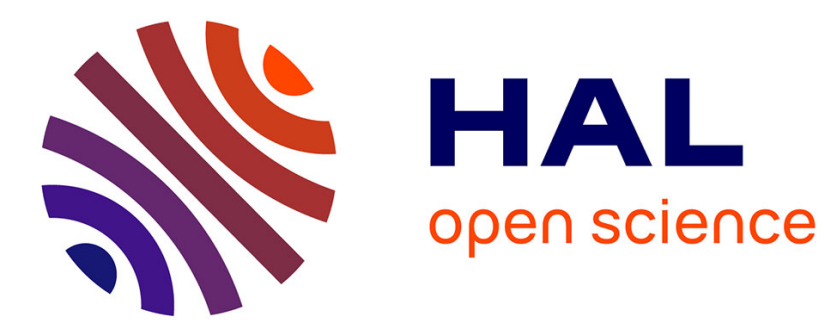

\title{
Les villes et ports de la Baltique, des interfaces aux portes de l'Europe
}

Nicolas Escach, Arnaud Serry

\section{To cite this version:}

Nicolas Escach, Arnaud Serry. Les villes et ports de la Baltique, des interfaces aux portes de l'Europe. Revue d'Etudes Comparatives Est-Ouest, 2015, Dossier: Habiter l'espace post-yougoslave, 46 (04), pp.229 - 263. 10.4074/S0338059915004088 . hal-01724134

\section{HAL Id: hal-01724134 \\ https://hal.science/hal-01724134}

Submitted on 6 Mar 2018

HAL is a multi-disciplinary open access archive for the deposit and dissemination of scientific research documents, whether they are published or not. The documents may come from teaching and research institutions in France or abroad, or from public or private research centers.
L'archive ouverte pluridisciplinaire HAL, est destinée au dépôt et à la diffusion de documents scientifiques de niveau recherche, publiés ou non, émanant des établissements d'enseignement et de recherche français ou étrangers, des laboratoires publics ou privés. 


\section{LES VILLES ET PORTS DE LA BALTIQUE, DES INTERFACES AUX PORTES DE L'EUROPE \\ Nicolas Escach, Arnaud Serry}

NecPlus | « Revue d'études comparatives Est-Ouest »

$2015 / 4 \mathrm{~N}^{\circ} 46$ | pages 229 à 263

ISSN 0338-0599

Article disponible en ligne à l'adresse :

https://www.cairn.info/revue-revue-d-etudes-comparatives-estouest1-2015-4-page-229.htm

\section{Pour citer cet article :}

Nicolas Escach, Arnaud Serry « Les villes et ports de la Baltique, des interfaces aux portes de l'Europe », Revue d'études comparatives Est-Ouest 2015/4 (N46), p. 229-263.

DOI $10.4074 / S 0338059915004088$

Distribution électronique Cairn.info pour NecPlus.

(C) NecPlus. Tous droits réservés pour tous pays.

La reproduction ou représentation de cet article, notamment par photocopie, n'est autorisée que dans les limites des conditions générales d'utilisation du site ou, le cas échéant, des conditions générales de la licence souscrite par votre établissement. Toute autre reproduction ou représentation, en tout ou partie, sous quelque forme et de quelque manière que ce soit, est interdite sauf accord préalable et écrit de l'éditeur, en dehors des cas prévus par la législation en vigueur en France. Il est précisé que son stockage dans une base de données est également interdit. 


\title{
LeS Villes eT PORTS de la BaltiQUe, DES INTERFACES AUX PORTES DE L'EUROPE
}

\author{
NICOLAS ESCACH ET ARNAUD SERRY
}

\author{
ATER en Géographie, Université de Versailles Saint-Quentin-en- \\ Yvelines ; Environnement Ville Société (EVS UMR 5600) \\ École normale supérieure de Lyon ; nicolas.escach@uvsq.fr \\ Maître de conférences en Géographie, UMR IDEES (6266), \\ Université du Havre ; serryarnaud@gmail.com
}

Résumé: Depuis 1989, les territoires baltiques se recomposent pour répondre aux impératifs qui ont suivi le démembrement de l'URSS. La chute du Rideau de fer a permis une intensification des contacts, la construction de liens fonctionnels et de réseaux institutionnels à l'échelle régionale. Paradoxalement, cette ouverture a posé la question d'une marginalisation de l'espace baltique dans une Europe politique en construction et dans des flux économiques mondiaux de plus en plus concentrés. Les acteurs riverains ont dû construire des antidotes à la "périphérisation », leurs territoires apparaissant certes trop petits pour s'intégrer directement dans des processus globaux mais assez grands pour espérer compter dans le futur de l'Europe. Être dans l'Union européenne tout en constituant une porte d'entrée peut avoir plus d'avantages que d'inconvénients. Des liens accrus avec la Russie, les États du partenariat oriental ou l'Asie sont aujourd'hui autant d'occasions d'ériger les villes et ports baltiques en carrefours entre la vieille Europe et un continent en pleine expansion économique et culturelle.

Mots ClÉs : mer Baltique, européanisation, mondialisation, réseaux, recomposition des niveaux. 


\section{INTRODUCTION}

La mer Baltique présente une situation géographique rare en Europe. Divisés par le Rideau de fer au cours de la guerre froide, les espaces qui en sont riverains sont longtemps restés des glacis (Mertelsmann \& Piirimäe, 2012). Les littoraux baltiques constituaient alors des marges ${ }^{1}$, bien loin des centralités continentales de l'empire soviétique et des zones de croissance d'une UE en pleine formation. La mer séparait des systèmes d'échanges qui lui tournaient le dos, à l'image du CAEM et de la CEE. De nombreuses bases militaires et villes fermées jalonnaient ses rives, créant jusqu'à aujourd'hui des configurations spatiales de no man's land à l'instar du quartier de Karosta à Liepāja. La chute du Rideau de fer (1989), l'élection de Lech Wałęsa en Pologne (1990) puis l'indépendance des États baltes (1991) accompagnent un retournement de perspectives. Les quatre pays riverains qui viennent de conquérir leur liberté cherchent à se détourner de la Russie et regardent vers l'Union européenne. La mer Baltique perd son statut d'angle mort pour devenir un relais. De Szczecin à Trieste, la mer Baltique et la mer Adriatique sont désormais susceptibles d'exercer un rôle de pont entre l'Est et l'Ouest de l'Europe (Dellenbrant, 1999). Les autorités locales et européennes prennent rapidement conscience de cette portée stratégique et multiplient les initiatives afin de couvrir l'espace baltique d'un tissu dense de réseaux d'acteurs issus de plusieurs niveaux géographiques. L'Union des villes de la Baltique (UBC) et l'Organisation des ports de la Baltique (BPO), créées en 1991, en sont les cas les plus emblématiques.

Le volontarisme des acteurs locaux, contraints de soigner les plaies du passé, le soutien par la suite des autorités européennes², soucieuses

1. Les coopérations entre les pays des deux blocs au nord de l'Europe sont alors plutôt rares même si des interactions peuvent exister, surtout pendant les périodes de « Détente » de la guerre froide (1962-1975, 1985-1991), comme en témoignent l'ouverture de la Conférence sur la sécurité et la coopération en Europe (1973) et la signature de la Convention d'Helsinki pour la protection du milieu marin dans la zone de la mer Baltique (1974).

2. L'adoption d'une initiative pour la région baltique par la Commission européenne le 10 avril 1996 et sa présentation aux chefs de gouvernement baltes lors de la conférence du Conseil des États de la mer Baltique à Visby, les 3 et 4 mai 1996, est une étape clé. La communication de la Commission de 1996 rappelle la nécessité d'une régionalisation baltique qui doit être le produit d'initiatives locales. Le soutien financier de la Commission doit répondre à une dynamique bottom-up, les acteurs rive- 
d'accompagner les élargissements nordiques (1995) et orientaux (2004), ont facilité l'apparition d'un espace transnational constitué de réseaux fonctionnels, institutionnels et de projets européens. Le terme d'espace transnational est ici à entendre comme système ouvert d'interactions et de réseaux établis à différents niveaux géographiques sur un espace aux limites floues par des acteurs issus de plusieurs États ${ }^{3}$ liés par une communauté d'intérêts ${ }^{4}$. L'espace transnational n'émerge que si l'ensemble des réseaux concernés s'inscrit dans un référentiel commun : les mêmes acteurs se retrouvent régulièrement au sein d'un nombre conséquent mais limité de structures coopératives et intègrent l'ensemble du système dans leurs réflexions et pratiques (stratégie de départ d'un réseau pour un autre, projets impliquant des synergies entre les différents forums...). Géographiquement, le caractère plus ou moins transnational d'un espace est assuré par ce positionnement relatif mais aussi par l'association d'une proximité topologique et d'un étalement géographique suffisant ${ }^{5}$, par des liens entre acteurs nombreux (masse critique) et efficients (régularité et intensité des liens, diversité des types d'acteurs impliqués, dimension concrète, utile et visible des résultats) et par une participation relativement « équitable » de l'ensemble des parties prenantes ${ }^{6}$.

Les études menées en sciences politiques et en géographie sur l'espace baltique depuis le début de la décennie 1990 l'ont souvent considéré isolément, concentrant les analyses sur l'émergence et l'évolution des interactions et échanges internes. Le processus de régionalisation a

rains pouvant espérer une assistance financière à condition qu'ils prennent l'initiative de monter des projets communs.

3. Dans cet article, l'espace transnational sera formé d'un ensemble continu d'au moins trois États dont deux a minima ne sont pas contigus.

4. La communauté d'intérêt ne signifie pas forcément que la coopération ait pour but une réalisation commune, ni même que les intérêts de chacun des acteurs soient similaires, mais ces derniers perçoivent ce qu'ils pourront retirer individuellement d'une mise en relation et surtout le risque qu'ils prendraient à rester isolés.

5. L'espace transnational étant constitué de réseaux, le niveau de connexité l'emporte sur la proximité physique. Il comprend des acteurs issus d'au moins trois États, souvent plus, dont deux a minima ne sont pas contigus. Ces conditions le distinguent de l'espace transfrontalier qui s'étudie à plus grande échelle.

6. Les acteurs, reflétant une variété de contextes professionnels, sont issus de différents États et s'inscrivent dans plusieurs niveaux géographiques. 
alors été défini comme le produit d'une déconstruction de la frontière ${ }^{7}$ est/ouest (Perko, 1996 ; Blanc-Noël, 2002 ; Kern \& Löffelsend, 2004). Cette position a contribué, dans un élan performatif, à une confusion entre posture programmatique et posture analytique. Un consensus entre scientifiques, élus et acteurs économiques s'est établi pour constater l'émergence d'une nouvelle région au sein de l'Union européenne (Joenniemi, 1991 ; Jervell, Kukk \& Joenniemi, 1992). Alors que la plupart des autorités riveraines diffusaient un discours de « retour à l'Europe » (Bayou \& Chillaud, 2012), les chercheurs multipliaient les séminaires et colloques sur l'unification de l'espace baltique, parfois dans des cadres très institutionnels ${ }^{8}$.

L'espace baltique a rarement été analysé dans le contexte de la recomposition des niveaux touchant les acteurs locaux depuis le début de la décennie 1980. Ceux-ci n'inscrivent plus seulement leur politique dans un cadre horizontal mais mènent également, avec plus d'autonomie vis-à-vis des États, des parcours entre et avec les niveaux géographiques. L'action territoriale s'inscrit nécessairement dans un double contexte d'européanisation politique ${ }^{9}$ et de mondialisation économique (Escach, 2014). Souvent appelée « politique des échelles », la politique des niveaux désigne la prise de conscience par les acteurs de la nécessité d'utiliser cet élargissement géographique comme ressource. Ceux-ci développent de véritables stratégies interterritoriales en mobilisant les connexions entre niveaux géographiques à bon escient afin de remplir les objectifs qu'ils se sont fixés.

Réseaux, espace transnational baltique et recomposition des niveaux contribuent à la même dynamique. L'existence d'un grand nombre d'interactions au niveau baltique s'explique en réalité par une situa-

7. Limite d'un État et de sa compétence territoriale, la frontière est par extension une limite séparant deux zones, deux régions ou même deux entités plus ou moins abstraites (BRUNET, 1992, p. 209).

8. Séminaire Zukunftsregion Ostsee à Travemünde en 1990, colloque The new Hansa à Kotka en 1990, conférences The Baltic Sea Area: A region in the making à Tallinn et The Baltic Sea Region: Conflict or Cooperation à Kiel en 1991 (Helmryd, 1993 ; NeumanN, 1998).

9. Elle revient, pour le cas particulier des villes, à prendre en compte l'opportunité mais aussi les nouvelles contraintes que l'adhésion suppose : en somme, à tirer les conséquences à l'échelle locale d'un élargissement géographique (HAMEDINGER \& WolfFHARDT, 2011). 
tion périphérique au sein des flux européens et globaux. Alors que les métropoles et ports d'importance de l'espace européen bénéficient d'une situation et d'une taille favorisant un ancrage direct à des dynamiques de niveaux plus élevés, la nécessité d'une médiation baltique, soit l'insertion au niveau baltique comme étape d'une intégration élargie, caractérise les acteurs riverains. La Baltique est un espace construit médian, articulant selon la logique hybride du « et » et non celle du « ou », acteurs, territoires et niveaux (Vergnaud, 2011). Les réseaux régionaux, facilitant le raccordement entre des espaces-limites, ouvrent sur un dépassement des horizons géographiques.

Si l'européanisation touche l'ensemble des autorités riveraines, celles-ci n'ont pas adopté une vision semblable de l'Europe et de ses limites géographiques. La Baltique orientale ${ }^{10}$ présente la particularité d'être une frontière de l'Union européenne tout en constituant une interface ${ }^{11}$ avec la Fédération de Russie et les États de la CEI. Les ports des États Baltes, notamment Riga pour les marchandises générales, Ventspils pour le pétrole et Tallinn-Muuga pour les pondéreux, étaient déjà pendant la guerre froide des gateways ${ }^{12}$ entre l'URSS, l'Europe et le reste du monde. La « persistance des territoires » semble spatialement bien plus importante qu'on ne l'avait imaginé (Serry, 2005). Les territoires de la Baltique orientale peuvent d'autant mieux profiter d'une position intermédiaire que leur résistance à la crise de 2008-2009 a été relativement élevée. La Pologne a même été considérée comme un bastion anti-crise de l'UE alors que les taux de croissance du PIB dans les États baltes affichaient à nouveau des valeurs imposantes dès 2011.

Notre intention n'est pas ici d'interroger la pertinence d'une grille est-ouest pour l'étude du degré de structuration de l'espace baltique,

10. Finlande, Estonie, Lettonie, Lituanie.

11. L'interface met en contact et en relation deux ensembles géographiques distincts générant des échanges entre les deux parties, une influence mutuelle et une exploitation de la différence par les acteurs.

12. Un gateway est une porte d'entrée principale d'un continent ou d'un pays. Les marchandises sont diffusées à partir du gateway selon des modes différents : le concept comprend la notion de "porte " (exemple : la porte maritime représentée par un port ou un ensemble de ports), de briques de transport mais au-delà, il intègre des composantes plus larges telles que la logistique et des propriétés territoriales étendues (exemples : l'attractivité, la compétitivité, l'industrie, la recherche, la gestion du foncier, l'acceptabilité). 
mais plutôt de déceler ce que l'espace baltique peut nous apprendre sur la recomposition des liens est/ouest à trois échelles d'analyse : 1'espace européano-russe, les liens avec les États de la CEI et les échanges transcontinentaux avec l'Asie orientale et la Chine. La clé d'entrée choisie est une comparaison entre les villes et les ports de la Baltique orientale, ces deux acteurs s'inscrivant dans des réseaux économiques et politiques à différents niveaux. L'espace baltique se prête particulièrement bien à une telle approche, le réseau urbain régional étant le produit d'une intégration fonctionnelle accrue entre des comptoirs portuaires à l'époque de la Hanse médiévale.

La démonstration que nous allons mener visera à questionner les effets de continuité historique ${ }^{13}$ pour un espace baltique soumis à un double processus d'européanisation et de mondialisation. Ces deux dynamiques ont souvent été perçues comme linéaires, et ne pouvant conduire qu'à un rattachement progressif de l'espace baltique aux hubs européens. En réalité, elles sont le résultat mouvant de trajectoires municipales et portuaires singulières sans cesse redéfinies par les acteurs locaux. En Baltique, l'interterritorialité est loin de se traduire par une échelle unique (l’Union européenne) ou par un horizon déterminant (l'ancrage occidental). Les élargissements géographiques dans lesquels les territoires riverains s'inscrivent sont protéiformes et combinent mutations, recompositions et persistances. Afin de mettre en évidence la situation intermédiaire de l'espace baltique, l'étude s'appuiera à la fois sur des sources qualitatives (données recueillies sur le terrain et entretiens principalement) et quantitatives (quatre bases de données) relatives aux réseaux de villes et aux trafics portuaires. Les limites de l'espace baltique, particulièrement délicates à définir, n'ont pas ici été fixées a priori. Dans le cadre des échanges portuaires, elles s'adaptent à un ensemble fonctionnel dynamique qui rassemble les principaux États riverains ${ }^{14}$ autour d'un avant-pays en partie partagé (la Northern Range). Les ports norvégiens, situés en mer du Nord, de l'autre côté des

13. En Baltique, l'héritage de la guerre froide est souvent perçu comme un frein, mais, en Baltique orientale, ses traductions spatiales peuvent être érigées en ressources : infrastructures intégrées de transport ferroviaire, contacts anciens vers l'est, langue commune pour certains acteurs... Ainsi, les nouvelles intégrations (dans les réseaux de l'Union européenne par exemple) s'ajoutent à une intégration ancienne réactivée (entre pays de l'ancien bloc de l'Est).

14. Allemagne, Pologne, Russie, États baltes, Finlande, Suède, Danemark. 
détroits danois, répondent à une autre logique. Pour les réseaux de municipalité, en revanche, l'aire d'éligibilité du programme INTERREG-B pour l'espace baltique ${ }^{15}$ constitue un référent incontournable puisque les acteurs souhaitant mener une démarche transnationale ont souvent recours au co-financement européen.

Après une première approche comparative des villes et ports de l'espace baltique, nous conduirons une analyse spatiale des réseaux portuaires et municipaux en Baltique, avant de mettre en évidence, dans le cas de la Baltique orientale, le rôle de ces interactions transnationales dans une intégration à des dynamiques plus larges (liens avec la Russie, la CEI et l'Asie).

\section{UNE APPROCHE COMPARATIVE ENTRE VILLES ET PORTS DANS LE CADRE BALTIQUE}

\subsection{LE COMPTOIR HANSÉATIQUE, UNE TRADITION BALTIQUE DE VILLE-PORT}

La plupart des grands ports régionaux ne sont pas de très grandes villes comme le montre la Figure 1. Saint-Pétersbourg constitue à ce titre une exception avec une population de 4502991 habitants et un trafic de 60 millions de tonnes en 2011 (Escach \& Serry, 2013), soit la première ville et le deuxième port (en volume) du littoral baltique. Les autres grands ports riverains comme Klaipèda, Ventspils, Gdańsk, Gdynia, sont en réalité des villes de taille moyenne (moins de 500000 habitants).

Les villes intégrées au sein de l'espace baltique répondent au modèle de la ville moyenne portuaire, qui était déjà celui de la cité hanséatique au Moyen Âge. La Hanse est une association de marchands dont la genèse est à trouver dans une première guilde de commerçants allemands formée vers 1161 à Visby sur l'île de Gotland. Le duc de Saxe, Henri de Lion, signe à cette époque l'accord d'Artlenburg qui permet aux marchands allemands, originaires de Lübeck, de Saxe ou de Westphalie, d'étendre leurs activités à parts égales avec les Gutes. L'association vise à déve-

15. Le programme INTERREG « Espace baltique » comprend les pays riverains européens dans leur ensemble, à l'exception de l'Allemagne représentée par les seuls Länder de Berlin, du Brandebourg, de Brême, du Mecklembourg-PoméranieOccidentale, du Schleswig-Holstein et de la Basse-Saxe (Regierungsbezirk Lüneburg). La Norvège, la Biélorussie et les régions septentrionales et orientales de la Russie sont également associées au programme. 
lopper le commerce maritime dans la région, sur des bases sûres, en luttant contre la piraterie (Champonnois, 2002). En 1241, le pivot des flux maritimes se déplace à Lübeck suite à l'alliance signée avec Hambourg. La ville connaît un développement rapide et s'impose comme une étape indispensable sur la route vers l'est, tant pour les marchandises que pour les colons et les mercenaires. En 1259, les cités de Wismar et de Rostock rejoignent l'association. En 1280, le blocus contre Bruges apporte à la Hanse un pouvoir quasi hégémonique en Europe du Nord. Peu à peu, la Hanse des marchands se transforme en Hanse des villes, rassemblant des cités libres qui accumulent les privilèges en Angleterre, en Flandre ou Norvège (Dollinger, 1964; Perchoc, 2006). En 1356, la première diète (Hansetag) tenue à Lübeck réunit l'ensemble des villes de la Hanse. La Hanse germanique s' affirme alors progressivement comme une puissance militaire (Escach \& Serry, 2013).

FIGURE 1

Les ports et les villes portuaires en mer Baltique en 2011

a. Trafic des ports

de la mer Baltique en 2011

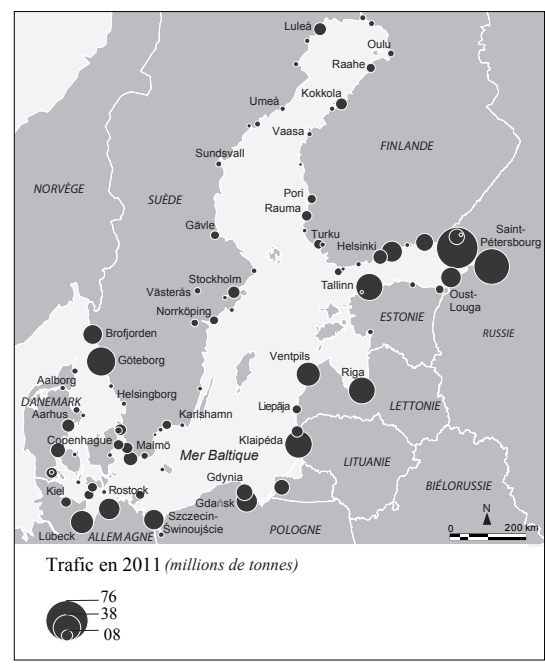

b. Les villes portuaires de la mer Baltique en 2011

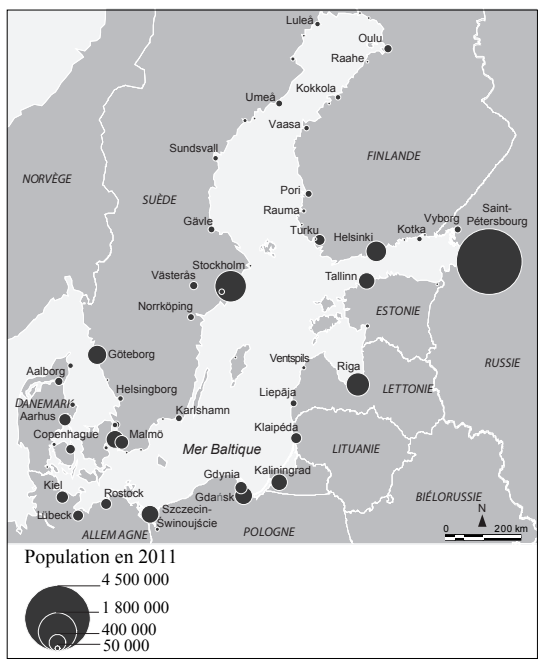

Sources : a) European Sea Ports Organization, Eurostat et sources portuaires diverses; b) Eurostat et World Gazetteer (C Escach, Serry, 2013

Les routes commerciales hanséatiques se présentent pour la plupart sous la forme de longitudinales est/ouest. La première voie marchande relie dès le XII ${ }^{e}$ siècle Novgorod à Lübeck en passant par Visby et permet l'acheminement de bois et de fourrures de Russie. Un approvision- 
nement en ambre, en lin et en bois est également assuré par des échanges intenses entre Vitebsk et Visby via Riga, le long de la Düna (Daugava). Entre 1250 et 1350, un troisième itinéraire, plus méridional, s'ouvre entre les villes de la Vistule et l'Allemagne du Nord par le port de Dantzig. Même si Lübeck continue de concentrer la plupart des marchandises à destination de l'Europe du Nord, Dantzig et Visby s'imposent progressivement comme des pivots secondaires, respectivement pour l'angle sud-est et l'angle nord-est de la Baltique entre 1350 et 1500.

Les villes hanséatiques présentent encore aujourd'hui de fortes similarités : une architecture gothique en brique rouge, des maisons avec pignons à échelons, la présence de quais anciens à l'image des Schlachte de Brême sur lesquels s'amarraient les anciens Kogge, les bateaux utilisés par les commerçants allemands. Des motifs régionaux sont visibles dans chacune d'entre elles : des Marienkirche, symboles de la ferveur religieuse des Hanséates contre le pouvoir épiscopal, aux statues de Roland rappelant l'importance des libertés municipales acquises sur les pouvoirs temporels. L'ensemble fait l'objet d'une intense patrimonialisation, comme en témoigne l'existence de circuits touristiques ou de croisières sur le thème des « perles de la Hanse ». De nombreuses boutiques et entreprises instrumentalisent à des fins publicitaires le mot « Hanse » car celui-ci reste associé à un ensemble de valeurs positives : la confiance, la fiabilité, la modestie, l'ouverture.

\subsection{ViLLES ET PORTS BALTIQUES, UNE SITUATION PÉRIPHÉRIQUE COMMUNE}

Villes et ports de la Baltique partagent donc un héritage historique commun mais aussi une situation géographique commune. Leur insertion au sein des flux mondiaux et européens est inégale et indirecte.

Ayant acquis une configuration spécifique dans l'économie maritime mondiale pour des raisons idéologiques et géographiques (la profondeur dans les détroits danois limite l'accès à des navires d'un tirant d'eau inférieur à 15 mètres), la mer Baltique reste aujourd'hui à l'écart des grandes routes maritimes qui desservent les ports pivots de l'organisation circumterrestre. Les ports de la Northern Range ${ }^{16}$ se sont rapidement imposés comme des hubs pour l'ensemble des ports baltiques. Même s'il n'est pas situé en mer Baltique, le port d'Hambourg n'en constitue pas moins le

16. La rangée nord-européenne est comprise comme un ensemble de ports s'étirant du Havre jusqu'à Hambourg et servant de façade maritime à l'Europe rhénane. 
pivot des échanges maritimes notamment dans le domaine du conteneur (Debrie, Eliot \& Soppé, 2005). En 2015, $88 \%$ des ports baltiques ${ }^{17}$ qui traitent des conteneurs entretiennent au moins une liaison avec Hambourg. Pour $80 \%$ de ces ports reliés à la métropole nord-allemande, Hambourg est l'une des trois premières destinations en nombre de liaisons.

L'ouverture économique des ports baltiques s'est paradoxalement manifestée par la rétraction de leur avant-pays qui se concentre de plus en plus sur les ports de la rangée nord-européenne. Hors liaisons avec la rangée nord-européenne, dominent des relations intra-baltiques. Ces dernières, qui utilisent notamment des navires rouliers et des ferries, marquent nettement le paradoxe baltique : à l'heure où l'économie baltique se mondialise, son transport se régionalise. Ce phénomène est renforcé par les politiques des armements. Second grand opérateur nordique, la société danoise DFDS a repris successivement Lisco (Lituanie) en 2001 et Latline (Lettonie) en 2003. Scandlines, déjà présente sur certaines lignes baltes, a repris par sa branche allemande l'activité fret d'Esco (Estonie), devenue Scandlines Estonia. Ces armements passés sous contrôle danois ou allemand se consacrent essentiellement aux liaisons intra-baltiques de services mixtes ou fret. Dans le même ordre d'idées, des opérateurs occidentaux se sont installés sur la rive balte, comme le manutentionnaire hambourgeois HHLA qui a participé à l'extension des terminaux à conteneurs du port de Saint-Pétersbourg. Par ailleurs, les autorités portuaires baltes s'efforcent d'attirer des investisseurs russes. Ainsi, l'entreprise pétrolière Lukoil a participé à la modernisation du terminal pétrolier de Ventspils désormais géré par le groupe international Vitol. De son côté, l'un des leaders russes dans la production d'engrais, la société UralChem possède désormais $51 \%$ des actions du nouveau terminal Riga Fertilizer Terminal LLC (RFT) du port de Riga. Ces exemples soulignent, si besoin est, qu'il ne faut pas confondre les stratégies géopolitiques des États et la stratégie des acteurs privés qui cherchent à diversifier leurs itinéraires et à conquérir des positions au-delà des frontières de la Russie (Thorez, 2011).

La hiérarchisation portuaire née du système de transbordement à partir de ports principaux (hubs) tient donc la mer Baltique dans une position que l'on qualifie souvent de secondaire. La desserte maritime

17. Il s'agit ici des ports riverains de la mer Baltique (Allemagne, Pologne, Russie, États baltes, Finlande, Suède, Danemark). 
en marchandises diverses s'opère en grande partie par des lignes de feeders ${ }^{18}$. La Baltique n'est pas suffisamment attractive pour les escales directes des navires transocéaniques ${ }^{19}$ : situation en cul-de-sac et fermeture par les détroits danois, faiblesse des volumes, fragmentation des escales réparties sur des distances rapprochées en sont les principales causes. La Baltique est donc essentiellement desservie par un réseau qui se rattache indirectement au réseau maritime mondial via les grands ports européens. Au départ des ports de la rangée du nord, les rotations des feeders sont soit circulaires - avec desserte de quelques ports dont, très souvent, Saint-Pétersbourg -, soit directes vers un ou deux ports.

La situation périphérique des ports baltiques renvoie à la configuration des villes riveraines. À l'exception de Saint-Pétersbourg, l'espace baltique compte peu de très grandes villes. La puissance de la métropole russe est d'ailleurs absolue puisque, dans leur ensemble, les villes de Stockholm, Helsinki, Copenhague, la Triville (Gdańsk-Gdynia-Sopot), Riga et Tallinn n'atteignent pas la taille de la ville fondée par Pierre le Grand ${ }^{20}$.

Les statistiques des régions métropolitaines d'Eurostat nous aident à estimer le poids des métropoles baltiques au sein de l'Union européenne élargie. Si l'on se fonde sur la définition de l'espace baltique proposée par le programme INTERREG IV-B, en 2014, les 45 régions métropolitaines définies par Eurostat et situées en Baltique ${ }^{21}$ rassemblent seulement $16 \%$ de la population des 278 aires métropolitaines des 28 pays de l'Union européenne auxquels s'ajoutent la Norvège et la Suisse.

18. Le feeder est un navire de petit tonnage, permettant l'éclatement, sur différents ports à courte distance, de conteneurs : les ports secondaires ou à l'écart des grandes routes sont desservis à partir des hubs où s'opère le transbordement direct de navire à navire (entre le navire-mère et le feeder).

19. Un port comme Gdańsk, qui figure depuis mai 2011 sur les itinéraires du service océanique de l'armement Maersk, fait figure d'exception (EsCACH \& SERrY, 2014).

20. http://www.voxeurop.eu (consulté le 15/07/2014).

21. Les régions métropolitaines des Länder allemands concernés par le programme INTERREG IV-B sont : Berlin, Hambourg, Schwerin, Neubrandenburg, Rostock, Lübeck, Kiel, Flensburg, Brême, Bremerhaven; au Danemark : Copenhague, Aarhus, Odense, Aalborg ; en Suède : Stockholm, Göteborg, Malmö, Uppsala; en Finlande : Helsinki, Turku, Tampere; en Estonie : Tallinn; en Lettonie : Riga; en Lituanie : Vilnius, Kaunas; en Pologne : Varsovie, Łódź, Cracovie, Wrocław, Poznań, Gdańsk, Szczecin, Bydgoszcz - Toruń, Lublin, Katowice, Białystok, Kielce, Rzeszów, Opole, Częstochowa, Radom, Bielsko-Biała, Tarnów; en Norvège : Oslo, Bergen. 
Elles représentent 47,7 millions d'habitants. Cela est très peu en comparaison du réseau urbain allemand dans son ensemble qui compte $18 \%$ de la population des régions métropolitaines européennes. Cependant, la définition baltique institutionnelle comprend l'ensemble des régions métropolitaines polonaises et une partie non négligeable des régions métropolitaines allemandes, ce qui gonfle les chiffres puisqu'elles comptent respectivement pour $40 \%$ et $25 \%$ de la population des régions métropolitaines baltiques en 2014. La prise en compte des seules régions riveraines de la mer Baltique, et donc une définition bien plus stricte de l'espace baltique, aboutit à des chiffres bien plus bas. Les 16 régions métropolitaines du littoral baltique ${ }^{22}$ ne représentent alors pas plus de $5 \%$ des habitants des régions métropolitaines européennes.

De même, peu de villes baltiques émergent dans les classements identifiant les villes globales ou même les villes de rang européen à partir d'indicateurs agrégés. Un indice des villes globales parmi les plus connus est l'indice du $\mathrm{GaWC}^{23}$, réalisé pour la première fois par Richard G. Smith et Peter J. Taylor en 1998 à l'Université de Loughborough. Selon la version de 2010, Varsovie est considérée comme une ville mondiale de type alpha, Berlin, Hambourg, Stockholm, Copenhague, Oslo et Helsinki de type bêta alors que les trois capitales baltes se trouvent dans la catégorie gamma ${ }^{24}$. La ville de Riga est la seule des trois capitales baltes à rejoindre la catégorie bêta dans le classement de 2012. Ces éléments convergent avec des travaux récents de la DATAR. Une étude coordonnée par Armand Frémont dans le cadre d'une mise en réseau des villes normandes propose une carte intéressante des villes européennes (Frémont, 2011). Varsovie, Stockholm et Berlin sont considérées comme des «métropoles européennes majeures », Copenhague, Helsinki, SaintPétersbourg, Minsk et Hambourg comme des « grandes villes d'importance européenne » et Riga et Göteborg comme des « grandes villes à potentiel européen $»^{25}$. De même, une synthèse de la DATAR publiée en

22. Rostock, Lübeck, Kiel, Flensburg, Copenhague, Aarhus, Odense, Aalborg, Stockholm, Malmö, Helsinki, Turku, Tallinn, Riga, Gdańsk, Szczecin.

23. Réseau d'étude sur la mondialisation et les villes mondiales.

24. Le GaWC classe les villes mondiales en trois groupes : villes alpha, villes bêta, villes gamma.

25. Voir la cartothèque du site Normandie métropole : http://normandie-metropole. fr (consulté le 24/07/2014). 
2012 ne retient que Berlin, Oslo, Copenhague, Stockholm et Helsinki dans le groupe des « métropoles très diversifiées » (Halbert et alii, 2012).

Les ports et les villes baltiques ne constituent donc pas des relais des grands flux mondiaux et européens mais plutôt un espace secondaire d'échanges. Une telle situation géographique est habituellement associée à une position d'infériorité, à une contrainte dont les acteurs locaux chercheraient à échapper, notamment pour se rapprocher du centre (Browning, 2005). Cette théorie a été contestée par Noel Parker pour qui un espace périphérique peut tout à fait exercer un rôle très important sur la scène internationale. La position périphérique place les acteurs dans une perpétuelle incertitude. Celle-ci constitue un fondement de leur action car l'appartenance de leur territoire à des niveaux plus élevés n'est jamais assurée. Elle leur permet d'adopter une démarche proactive. À l'inverse, le centre a besoin de beaucoup d'énergie pour veiller à ce que les marges ne le concurrencent pas. Les acteurs des espaces périphériques ont en main un large panel d'options qui peuvent constituer autant d'alternatives à un ralliement au centre. Cette variété de choix représente une différence majeure entre le centre et les périphéries (Parker, 2000). La capacité des autorités urbaines et portuaires baltiques à innover et à créer des cadres d'action au niveau baltique pour entrer dans les réseaux européens et mondiaux est nourrie par la peur de ce qu'ils conçoivent comme un risque de marginalisation. Il s'agit de transformer une situation a priori défavorable et de réagir à des évènements d'envergure continentale (à l'image de la chute du Rideau de fer) pour faire de ces crises des opportunités.

\section{L'ESPACE BALTIQUE COMME ESPACE INTERMÉDIAIRE : ÉRIGER LA PÉRIPHÉRIE EN RESSOURCE}

Selon notre hypothèse, les autorités municipales ou portuaires baltiques $^{26}$ visent à transformer la position d'entre-deux des territoires riverains en ressource territoriale ${ }^{27}$. À cette fin, elles utilisent l'espace

26. Pour les bases de données de ports, la délimitation de l'espace baltique choisie correspond aux territoires riverains de la mer Baltique (exclusion de la Norvège).

27. Par « ressource territoriale », nous entendons une propriété du territoire (y compris sa situation) que les acteurs révèlent, à laquelle ils attribuent une valeur et dont ils souhaitent tirer parti dans le cadre de leurs discours, représentations et pratiques spatiales. 
intermédiaire baltique et les avantages qu'il offre (forums de coopération, réseaux institutionnels, projets européens). Les divergences et rivalités entre ports et villes de la Baltique sont intenses. La concurrence est cependant surmontable lorsque l'intérêt commun se situe à un autre niveau géographique. La constitution d'un groupe est alors nécessaire pour renverser une situation a priori défavorable. Ce handicap est lié à un ordre de grandeur (faible nombre d'habitants, activités peu diversifiées) ou à une localisation (éloignement d'une frontière ou du littoral, faibles opportunités d'internationalisation), voire à un croisement des deux facteurs.

\subsection{Mesurer L'INSERTION DES VILLES Et PORTS AU SEIN DE L'ESPACE BALTIQUE}

Afin d'évaluer l'utilisation différenciée des réseaux baltiques par les acteurs riverains, encore fallait-il mesurer leur intégration au sein de ceux-ci. Nous avons pour cela utilisé des bases de données rassemblant des ports et des municipalités baltiques pour les années 2013, 2014 et 2015 .

En ce qui concerne les ports baltiques, trois bases de données principales ont été mobilisées à partir de différentes sources ${ }^{28}$. Une première base $\left(\mathrm{BD}_{\mathrm{p} 1}\right)$ a rassemblé l'ensemble des ports baltiques intégrés dans des lignes ro-ro $0^{29}$ et ro-pax en $2013^{30}$. Une seconde base $\left(\mathrm{BD}_{\mathrm{p} 2}\right)$ a regroupé les ports disposant de terminaux conteneurs ${ }^{31}$ de l'espace baltique en 2014 et les lignes correspondantes mi-2015. Ces deux bases ont permis de mesurer l'intégration au sein de réseaux économiques des ports baltiques. Une troisième base $\left(\mathrm{BD}_{\mathrm{p} 3}\right)$ a évalué la participation

28. Baltic Ro-ro \& Ferry Yearbook 2013, Baltic Transport Journal, sites des ports baltiques, données recueillies sur le terrain pour les bases BDp1 et BDp2. Site Internet INTERREG IV-B et sites des réseaux institutionnels pour la base BDp3.

29. Ro-ro est une abréviation utilisée pour roll on-roll off, technique de manutention horizontale des navires. Les véhicules sur roues empruntent une rampe d'accès aux portes arrière ou latérales et des rampes intérieures fixes ou mobiles vers les différents ponts. On appelle ro-pax de l'anglais roll on-roll off-passenger-ship, le navire roulier mixte capable de transporter des marchandises et des passagers.

30. Soit 72 ports au total.

31. Soit 43 ports au total. 
des ports baltiques à des réseaux politiques, qu'il s'agisse de réseaux institutionnels ${ }^{32}$ ou de projets européens ${ }^{33}$ pour la période 2007-2013.

Pour le cas des municipalités baltiques ${ }^{34}$, nous avons élaboré une base de données $\left(\mathrm{BD}_{\mathrm{v} 1}\right)$ rassemblant des réseaux de deux natures différentes pour l'année 2013 : des réseaux institutionnels ${ }^{35}$ et des projets INTERREG IV-B ${ }^{36}$. Plusieurs critères ont conduit à la sélection des réseaux ${ }^{37}$ de la base de données. Le caractère transnational d'un réseau a été établi lorsque celui-ci associait des municipalités ${ }^{38}$ d'au moins trois États baltiques (y compris la Norvège, la Russie, la Biélorussie) dont deux au moins n'étaient pas contigus. Un État ne devait pas concentrer plus de $80 \%$ des membres d'un réseau donné ${ }^{39}$. Les réseaux retenus devaient aussi compter des municipalités parmi leurs membres, même si l'acteur municipal n'était pas exclusif ${ }^{40}$.

32. Baltic Sea Action Group, BPO, Cruise Baltic.

33. ACL, Baltic Master II, BGLC, BSR Innoship, BSR TransGovernance, Cleanship, EWTC II, SCANDRIA, SMOCS, TRANSBALTIC, TransBaltic EXT.

34. Pour les bases de données de municipalités, nous délimitons l'espace transnational baltique conformément au programme européen INTERREG IV-B : 1'Estonie, la Lettonie, la Lituanie, la Pologne, la Suède, le Danemark, la Biélorussie, la Norvège et une partie des régions allemandes et russes.

35. La liste des réseaux institutionnels, n'associant que des municipalités ou rassemblant des municipalités et d'autres types d'acteurs, a été établie à partir de l'annuaire 2013/2014 des organisations internationales de l'Union des associations internationales (référence : Union of International Associations (2013), Yearbook of International Organizations 2013/2014, Leiden/Boston, Brill, 1424 p.).

36. La liste des projets INTERREG IV-B (2007-2013) est publiée sur le site du programme.

37. Dans l'article, le terme réseau sera utilisé pour désigner à la fois des réseaux institutionnels et des projets européens.

38. Il était délicat de concevoir un seuil autre que la population (ici un minimum de 10000 habitants) pour identifier les municipalités baltiques car la définition de la ville n'est pas unifiée au sein des espaces riverains de la Baltique : certains États retiennent un seuil statistique ou historique alors que d'autres considèrent la ville comme un statut défini par la loi, le gouvernement central ayant le pouvoir de conférer des attributs urbains.

39. Ce critère nous a amenés à exclure le réseau Baltic Sea Challenge puisque $80,6 \%$ des villes membres étaient situées en Finlande.

40. Les bases de données ont été construites à partir des acteurs municipaux, c'està-dire des élus ou employés de l'administration centrale ou des départements de la mairie de la ville concernée. 
La base de données initiale a rassemblé 4 réseaux institutionnels de municipalités ${ }^{41}, 12$ réseaux institutionnels associant des municipalités $^{42}$ et 47 projets européens INTERREG IV-B, soit 63 réseaux et projets transnationaux baltiques au total ${ }^{43}$.

Le choix de ne retenir que les réseaux institutionnels et les projets européens ne doit pas faire oublier qu'ils ne constituent qu'une partie des interactions baltiques. Ils s'inscrivent dans une multitude de formes d'échanges plus ou moins structurés impliquant différentes temporalités (jumelages, rencontres multilatérales ou bilatérales informelles, coopérations ponctuelles). L'optimisation des ressources que les réseaux baltiques proposent dépend moins du rayonnement des municipalités que de celui des acteurs qui y travaillent. La différence entre une appréhension des réseaux de villes et une analyse des réseaux urbains réside bien dans le caractère intentionnel, volontariste et stratégique des rapprochements. En outre, l'adhésion à un réseau, parfois purement formelle, ne suppose pas forcément la participation à des activités concrètes. $A$ contrario, des résultats tangibles sont particulièrement difficiles à interpréter, si l'objectif est de distinguer la part de responsabilité du réseau par rapport à d'autres formes de synergie. Un certain flou se dégage donc des coopérations inter-municipales, ce qui a sans doute conduit à les étudier trop peu souvent.

L'étude des quatre bases de données constitue le fil directeur d'une argumentation qui s'appuie sur trois ressorts principaux :

41. BaltMet, KIMO, Hanse des temps nouveaux, Union of Baltic Cities.

42. Ars Baltica, Baltic Local Agenda Forum 21 (BLA21F), Baltic Palette, Baltic Sail, Baltic Sea Action Group, Baltic Development Forum, Baltic Sea States Subregional Co-operation (BSSSC), Baltic Tourism Commission (BTC), EuRoB, Hanseatic Parliament, Healthy Cities Network Phase V, Social-Hansa.

43. Agora 2.0, BaltCICA, BaltFood, Baltic Biogas Bus, Baltic Bird, Baltic Fashion, Baltic Master II, Baltic Supply, Baltic. AirCargo. Net, Balticclimate, BaltMet Promo, BaSIC, BERAS Implementation, BGLC, BONITA, BSLN, BSR InnoShip, BSR Quick, BSR TransGovernance, BSR InnoReg, CleanShip, COHIBA, CoolBricks, Eco-Region, Egoprise, EWTC II, FM, IBI Net, JOSEFIN, More Baltic Biogas Bus, Longlife, NECL II, New Bridges, One BSR, Parks and Benefits, PEA, PRESTO, PURE, RB21T, RBGC, SCANDRIA, Science Link/Link1, Submariner, Trans in Form (TiF), Urb. Energy, Urban Creative Poles, Waterpraxis. 
Le filtrage ${ }^{44}$ des bases de données permet de dégager les municipalités et les ports les plus intégrés au sein des réseaux économiques, politiques et institutionnels baltiques et donc d'extraire un ensemble plus facile à représenter graphiquement et à analyser rigoureusement. Ce filtrage a été réalisé à l'aide du logiciel de statistiques $\mathrm{R}$.

La cartographie des données de la base principale et des bases filtrées sous Mapinfo offre une géographie des réseaux baltiques et l'identification de facteurs permettant de l'expliquer. Deux résultats ont fait l'objet d'une représentation cartographique : le degré d'intégration (nombre de réseaux/lignes par ville ou port) et le degré de coopération (nombre de réseaux/lignes entre deux villes ou ports).

Le croisement des bases de données avec d'autres sources plus qualitatives (données recueillies sur le terrain, entretiens, sources écrites) permet un recoupement des informations et a donné lieu à la mobilisation de nouvelles méthodes d'analyse (analyses de discours...).

\subsection{RÉALISER UNE GÉOGRAPHIE DE L'INTÉGRATION DES PORTS}

ET DES VILLES AU SEIN DE L'ESPACE BALTIQUE

D'un point de vue portuaire, les vingt dernières années ont été synonymes de croissance rapide et partagée des trafics surtout sur la façade orientale de la mer Baltique. Le trafic maritime dans la région a ainsi doublé au cours de cette même période. Celui-ci est désormais de plus de 800 millions de tonnes par an, composé à $42 \%$ de vrac liquide, $26 \%$ de vrac sec et $32 \%$ de marchandises diverses. Par ailleurs, les ports russes connaissent des taux de croissance très importants. Oust-Louga est ainsi devenu le premier port de la région en 2014 avec 75,7 millions de tonnes (Mt) devant Saint-Pétersbourg (61,2 Mt) et Primorsk (53,7 $\mathrm{Mt}$ ), autre nouveau port russe dans le golfe de Finlande. Le port de Saint-Pétersbourg a ainsi vu son trafic augmenter de $95 \%$ entre 2000 et 2014. La croissance des ports occidentaux est, quant à elle, moins rapide, ne dépassant pas les $30 \%$ sur la période 2000-2014.

44. Dans le vocabulaire statistique, un filtre permet de limiter l'affichage d'une base de données selon un critère déterminé (ici le nombre de réseaux par ville appelé aussi degré d'intégration). 


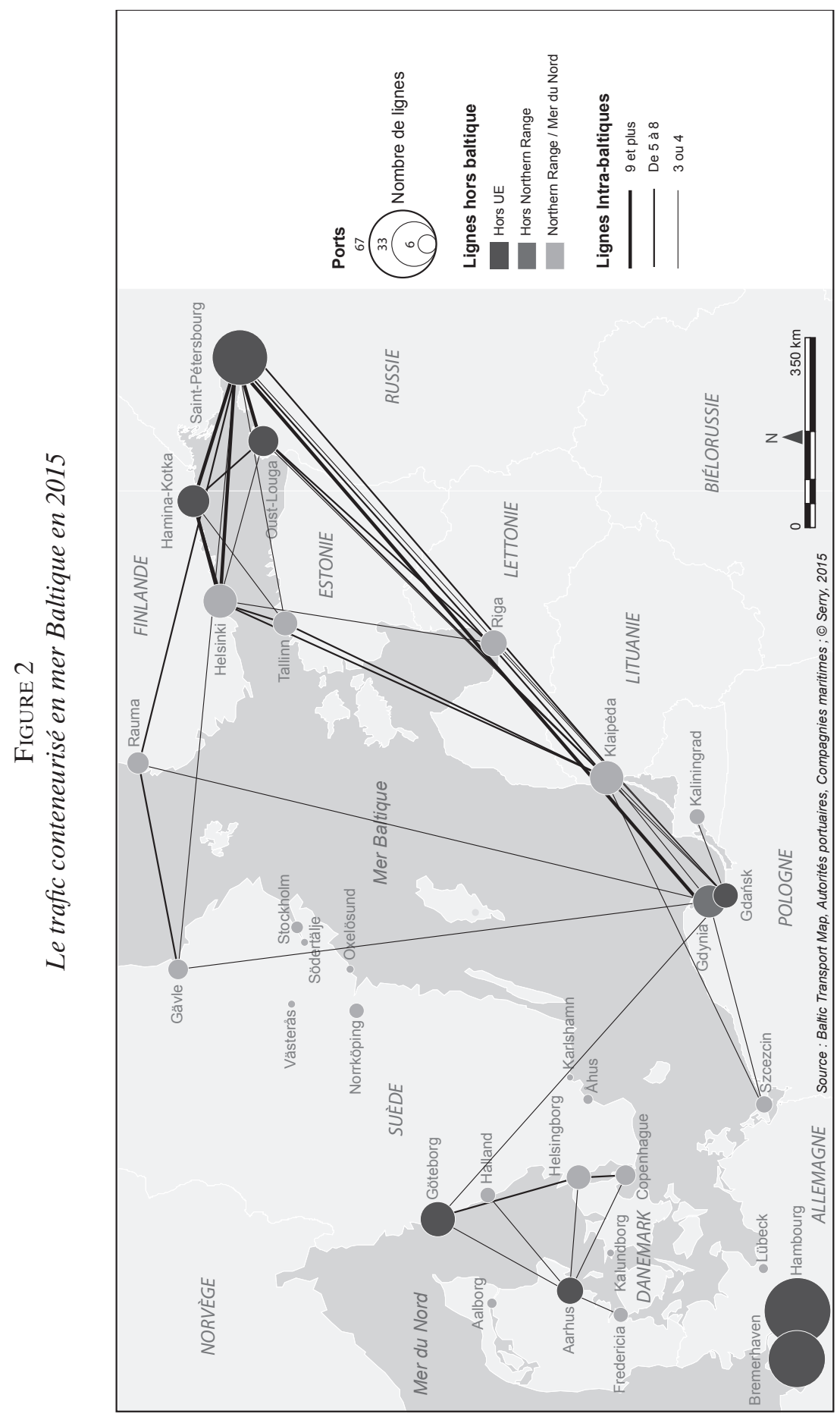


Les ports les plus intégrés au sein du trafic roulier (ro-ro et ro-pax) sont les ports du golfe de Finlande (Hanko, Helsinki, Hamina-Kotka, Saint-Pétersbourg); les lignes qui en partent sont néanmoins dirigées vers des ports baltiques et extra-baltiques (ports belges ou néerlandais). Ceux de la Baltique orientale (Riga, Ventspils, Klaipeda) et du littoral allemand (Travemünde, Lübeck, Kiel) présentent un profil régional plus évident avec des liaisons exclusivement tournées vers les autres ports riverains. À l'échelle baltique, la plupart des liaisons ne sont cependant pas transnationales mais locales notamment au sein de trois zones d'intégrations majeures : 1'Øresund, la Baltique méridionale (autour des littoraux suédois, polonais et lituaniens) et le golfe de Finlande. En 2013, sur 114 lignes roulières, un peu moins d'un tiers (30\%) étaient transnationales (lien entre deux zones), un quart (26\%) avaient pour destination des ports extra-baltiques ${ }^{45}$, alors que $44 \%$ des lignes reliaient deux ports d'une même zone transfrontalière.

Le trafic conteneurisé introduit une nette différenciation des activités maritimes et portuaires au sein de la mer Baltique (Figure 2). Les ports de la Baltique orientale comme Saint-Pétersbourg (48 lignes), Klaipèda (20 lignes), Helsinki (20 lignes) ou Gdynia (18 lignes) ainsi que des ports de l'Øresund comme Göteborg (20 lignes) et Aarhus (13 lignes) apparaissent comme les pôles majeurs. Sur la rive orientale de la mer Baltique comme dans les détroits danois, la mise en réseau intra-baltique des ports est évidente.

L'inscription des ports baltiques dans des réseaux institutionnels ${ }^{46}$ et des projets européens ${ }^{47}$ individualise Klaipèda ( 8 réseaux). Suivent les ports de Gdynia, Hambourg, Rostock (4 réseaux), puis Helsingborg, Helsinki, Karlshamn, Sassnitz, Stockholm, Tallinn et Turku (3 réseaux). Les ports de la Baltique orientale présentent donc un degré d'intégration particulièrement important mais sont aussi très bien reliés entre eux. Trois réseaux baltiques ${ }^{48}$ relient Stockholm et Helsinki, Tallinn et Helsinki, Turku et Helsinki ou encore Turku et Stockholm.

45. Norvège, Northern Range ou reste du monde.

46. Baltic Sea Action Group, BPO, Cruise Baltic.

47. ACL, Baltic Master II, BGLC, BSR Innoship, BSR TransGovernance, Cleanship, EWTC II, SCANDRIA, SMOCS, TRANSBALTIC, TransBaltic EXT.

48. Réseaux institutionnels et projets européens confondus. 
FIGURE 3

Les municipalités les plus impliquées dans les réseaux et projets baltiques en décembre 2013

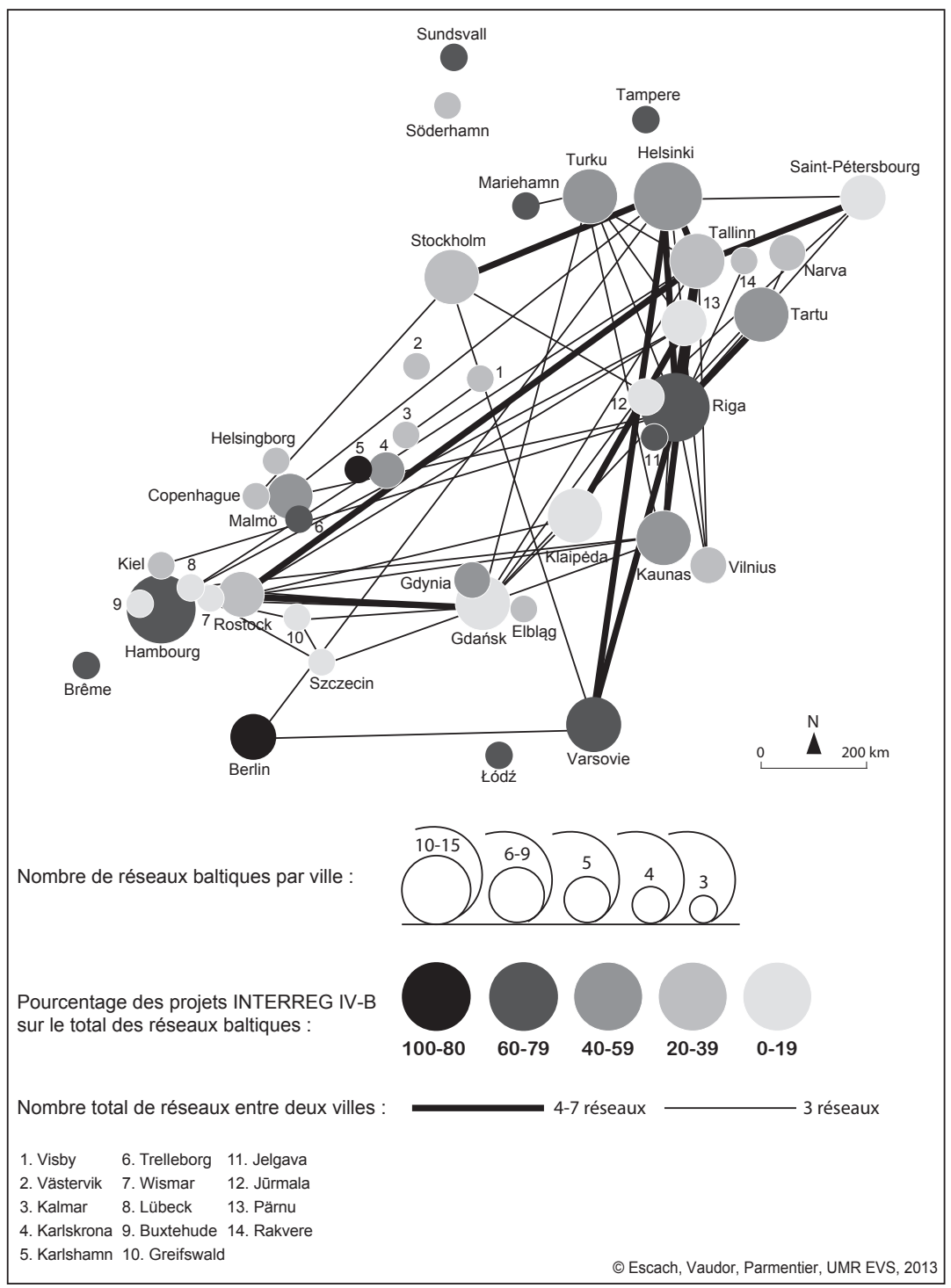

En ce qui concerne les réseaux et projets impliquant des municipalités, Riga (15 réseaux), Helsinki (12 réseaux) et Hambourg (10 réseaux) constituent les têtes de réseaux comme le montre la Figure 3. Suit un groupe de villes parmi lesquelles Tallinn, Tartu, Turku, Gdańsk, 
Kaunas, Klaipėda, Varsovie ou Stockholm. De manière générale, une ligne allant de Turku à Hambourg distingue une rive sud assez active (autour des pôles de l'Allemagne du Nord, du golfe de Finlande et de l'ensemble sud-est Kaliningrad/Gdańsk/Klaipeda) d'une rive nord discrète. Les capitales baltiques sont relativement bien représentées sauf Copenhague ( 3 réseaux) et Vilnius ( 4 réseaux). Elles semblent très bien reliées entre elles au niveau du golfe de Finlande (Helsinki, Stockholm, Tallinn, Riga). Ces capitales étatiques voire régionales présentent également un fort pourcentage de projets INTERREG parmi le nombre total de réseaux baltiques.

Trois constats peuvent être dressés à partir de cette analyse croisée :

Les liaisons roulières, particulièrement les ro-pax, ainsi que les réseaux de municipalités sont a priori considérés comme des vecteurs d'intégration transnationale. Leur géographie cache en réalité une sousrégionalisation de l'espace baltique en trois ensembles d'intégration locale (l'Øresund, la Baltique méridionale, le golfe de Finlande).

Le golfe de Finlande et plus largement la Baltique orientale représentent deux zones particulièrement actives au sein des réseaux baltiques. Le déplacement du centre de gravité de l'espace baltique vers l'est semble avoir étiré ce que certains auteurs nommaient au début de la décennie 1990 la « banane bleue baltique $»^{49}$ ou la « petite Baltique » fondue dans la « grande Baltique » (Joenniemi \& Wæver, 1992 ; Helmryd, 1993).

Les autorités municipales et portuaires de la Baltique orientale sont particulièrement actives en mer Baltique ce qui ne veut pas dire que l'espace baltique constitue la priorité absolue dans la perspective d'un déploiement international. La Finlande et les trois États baltes sont en effet caractérisés par une suprématie des capitales étatiques dans l'ancrage transnational avec un rayonnement important d'Helsinki ou Riga. La participation à des réseaux baltiques s'accompagne d'une stratégie de partenariat étendue à des pivots européens et internationaux.

49. La « banane bleue baltique » dessine un large espace de Hambourg à Helsinki. 


\section{LA BALTiQue ORIENTALE : UTILISER L'ENTRE-DEUX COMME UNE RES- SOURCE TERRITORIALE}

La Baltique orientale présente plusieurs caractéristiques qui justifient d'en faire une étude de cas plus approfondie. Le dynamisme des échanges commerciaux et le rayonnement des acteurs municipaux restent concentrés dans un petit nombre de localités, souvent susceptibles d'étendre leur influence dans un espace plus large que les seuls territoires baltiques. Se positionner au sein de l'espace transnational baltique permet alors d'atteindre des partenaires peu accessibles directement. Les ports et municipalités finlandaises et baltes ont également comme point commun de se trouver à la périphérie de l'Union européenne mais à proximité d'ensembles urbains et portuaires hors UE très imposants. À mesure que la distance avec l'isthme européen croît, l'attractivité de pôles à proximité de la frontière Schengen peut être grandissante. Le golfe de Finlande a ainsi connu un redéploiement du trafic russe à travers la construction ex-nihilo de nouveaux ports à l'instar de Primorsk, devenu le premier port régional à la fin des années 2000. Au niveau des municipalités, il existe 164 villes de plus de 100000 habitants en Russie et 13 en Biélorussie, certes situées pour beaucoup à distance de la mer Baltique, contre huit en Finlande et une seule en Lettonie et Estonie ${ }^{50}$. À proximité de la frontière avec les États baltes, les municipalités de Saint-Pétersbourg (5 millions d'habitants en 2014), de Kaliningrad (450 000 habitants) ou de Grodno (350 000 habitants) constituent des pôles d'attraction majeurs.

\subsection{La BaLtique orientale, un pont Vers la Russie?}

La Baltique orientale a connu une longue période d'occupation russe : la Finlande est devenue indépendante en 1917 et les États baltes le sont redevenus en 1990-1991. L'influence russe sur l'est de la Baltique est donc encore prégnante.

La Baltique orientale constitue un espace de collision entre les aires d'influence de l'Union européenne et de la Russie. Elle reste une limite fortement chargée en symboles et représentations. Mais, contrairement aux considérations habituelles, elle ne juxtapose pas différents maillages ou réseaux. Ces derniers sont en grande partie hérités de l'époque sovié-

50. Sites statistiques nationaux pour la Russie et la Biélorussie, audit urbain d'EUROSTAT pour les États membres de l'UE. 
tique. Les transports contribuent, de ce fait, à remettre en question les frontières. La discontinuité engendre ici des processus d'organisation spécifique de l'espace, un espace gateway, dans le sens de région polarisant des flux très importants avec un impact sur le territoire environnant. Les marchandises sont acheminées à partir de cette région gateway dans un milieu différent et par des modes différents. De nombreux indicateurs prouvent que, passés les troubles des premières années d'indépendance, de nouvelles relations commerciales se sont développées sur des fondations issues d'anciennes accointances (Serry, 2013).

Le rôle des ports de la Baltique est primordial dans la desserte de la Russie et d'autres pays de la CEI, notamment pour l'acheminement des conteneurs vers les zones de consommation : le cœur économique de la Russie (région de Moscou) et quelques destinations plus lointaines. Les hydrocarbures (russes) occupent une place prépondérante dans les flux à l'exportation, principalement dans les ports de la Baltique orientale. En 2010, le transit de marchandises de la CEI représentait $42 \%$ du trafic du port lituanien de Klaipéda et près de $80 \%$ des trafics portuaires lettons ou estoniens. Après avoir profité à Ventspils (port traditionnel d'exportation des hydrocarbures soviétiques), le trafic pétrolier a fait le bonheur du port de Tallinn. Depuis 1999, la Russie opère un resserrement progressif des terminaux d'exportation vers le territoire national, comme pour de nombreux autres produits en transit (Le Bourhis, 2009).

Le trafic de transit s'accompagne de multiples contraintes et pressions pour les États concernés que l'on pourrait résumer en six ensembles :

Pour la Russie, des contraintes économiques avec des frais de transit parfois élevés et extrêmement volatiles fixés par les États traversés.

Des contraintes stratégiques avec une forte dépendance des ports baltes et la possibilité pour la Russie de « couper les vannes » comme ce fut le cas avec l'Ukraine en 2006.

Des contraintes techniques, notamment la persistance du réseau ferroviaire à écartement russe dans les États Baltes.

Des contraintes politiques avec l'entrée dans l'UE puis dans l'espace Schengen qui a engendré une modification des régimes douaniers à la frontière. Les autorités européennes ont également cherché à développer un axe méridien sud-nord (Rail Baltica, Via Baltica) qui ne porte pas pour l'instant une activité économique aussi intense que l'axe est-ouest. 
Des contraintes sociétales comme à Daugavpils où les trains remplis de wagons-citernes de pétrole transitent tous les jours vers les ports lettons.

Des contraintes environnementales, enfin, sur le parcours des oléoducs, des gazoducs ou des trains chargés d'hydrocarbures et dans les ports.

Les municipalités baltes et finlandaises ont également développé des liens avec la Russie toute proche. Helsinki, Tallinn et Saint-Pétersbourg coopèrent dans le cadre d'une stratégie intégrée du golfe de Finlande reprise dans plusieurs documents programmatiques. En 2011, les employés de la division des relations internationales de la municipalité d'Helsinki se sont rendus 30 fois à Saint-Pétersbourg et 25 fois à Tallinn, ce qui représente $40 \%$ des visites à l'étranger de l'année. La coopération est largement impulsée par la capitale finlandaise et relève autant du choix de raison que du choix de passion. La Finlande est directement affectée par la pollution engendrée par les activités russes, notamment celles de la métropole de Saint-Pétersbourg. En 2009, la deuxième ville de Russie et ses alentours ont été responsables de la moitié des décharges d'azote et des trois quarts des rejets de phosphore dans le golfe (OCDE, 2009). La proximité du port pétrolier de Primorsk provoque un trafic maritime très intense, responsable de fuites, voire d'un risque important de marée noire.

Les principaux liens entre villes russes et villes baltiques membres de l'Union européenne s'inscrivent dans le cadre de réseaux institutionnels et projets européens transfrontaliers de voisinage et partenariat $(\mathrm{ENPI})^{51}$. Ces derniers associent surtout des petites et moyennes villes, la partie méridionale de la Baltique orientale, notamment la région de Kaliningrad et la frontière avec l'Ukraine, étant nettement plus active que la partie septentrionale. Les liens avec la Russie sont donc particulièrement faibles au niveau du lac Peïpous et des frontières orientales des États baltes. Ces zones frontalières, loin d'être inactives, n'offrent pas encore un cadre privilégié pour une coopération institutionnelle, comme l'a montré Vincent Dautancourt, qui a travaillé sur la frontière entre l'Estonie et la Russie (Dautancourt, 2011). Il soulève le paradoxe

51. Liste des programmes entrant dans le cadre de l'ENPI (The European Neighbourhood and Partnership Instrument) : Programme Kolarctique-Russie, Programme Carélie-Russie, Programme Sud-est de la Finlande-Russie, Programme Estonie-Lettonie-Russie, Programme Lituanie-Pologne-Russie. 
d'un espace frontalier plutôt vide de coopération mais assez dense au niveau des activités et trafics informels, notamment autour du différentiel de prix de l'essence.

À la différence des transits est-ouest encore actifs sur un plan portuaire, les échanges entre municipalités intra-européennes et russes n'ont pas encore pu s'établir dans le cadre de réseaux structurés. Cela s'explique par des contraintes de quatre types particulièrement importantes et difficilement surmontables :

Des contraintes spatiales. Les frontières entre États baltes et Russie présentent un profil assez unique en Baltique. Tout d'abord, elles ont été réactivées pour la plupart très récemment : la dernière signature d'un traité frontalier bilatéral officialisant le tracé entre un État balte et la Russie remonte au traité entre l'Estonie et la Russie du 18 février 2014. Ce dernier prévoit un échange de 128,5 hectares de terre et 11,4 km² de surface aquatique dans le lac de Peïpous où seront installés des bornes et des itinéraires de navigation. Le coût d'installation de cette frontière devrait être de 26 millions d'euros. La difficulté à fixer la frontière dans cette zone provient également de la difficulté à borner un ensemble organisé par des traits physiques, la présence d'un immense lac notamment. La géographie des frontières en Baltique orientale est également singulière en raison de la présence de l'enclave de Kaliningrad. Enfin, à une autre échelle d'observation, les contraintes géographiques s'expriment par un redéploiement des intérêts européens et russes marginalisant sur un plan diplomatique et politique l'axe est/ouest. Alors que l'UE cherche à développer des connexions nord-sud (Via Baltica, Via Hanseatica), la Russie redéploie progressivement son trafic autour du golfe de Finlande (Richard, 2011).

Des contraintes interterritoriales. Les outils de l'UE ne permettent pas véritablement une intégration des voisinages et de la Russie au niveau des municipalités. Les projets ENPI et INTERREG IV-B s'avèrent plus efficaces pour une intégration des autorités régionales, notamment dans une perspective de coopération avec la Russie. Le programme Sud-est de la Finlande-Russie ne concerne quasiment exclusivement que des acteurs régionaux (Carélie du sud en Finlande, Oblast de Leningrad, ville d'État de Saint-Pétersbourg en Russie), ce qui explique la relative absence du golfe de Finlande et de Saint-Pétersbourg sur les cartes des projets ENPI. La coopération est nettement plus aisée au niveau régio- 
nal du fait d'une centralisation relativement forte côté russe. Les financements et autorisations doivent souvent remonter puis redescendre la cascade verticale traditionnelle (villes-régions-États), le tout dans un contexte très formaliste, ce qui rend la coopération d'autant plus difficile et laborieuse qu'elle est décentralisée. Au niveau des municipalités, la complexité administrative, la corruption et les chevauchements de pouvoir sont assez courants. Cette inadéquation des outils est également visible dans le secteur maritime. Les Autoroutes de la Mer, portées par l'UE et visant au développement d'un transport durable en favorisant un report modal de la route vers la mer, ne peuvent par exemple pas concerner un port russe, ce qui limite considérablement leurs potentialités dans la région.

Des contraintes institutionnelles et financières. Rappelons que la Russie ne coopère pas au sein de la politique européenne de voisinage (PEV) qu'elle a refusé de ratifier mais fait l'objet d'un partenariat stratégique autour de quatre espaces communs (espace économique, espace de liberté, de sécurité et de justice, espace de sécurité extérieure, espace de recherche et d'éducation). Yann Richard évoque une impossible coopération transfrontalière entre la Russie et l'UE (Richard, 2011). Le développement de relations plus intenses au-delà de la limite de l'UE bute tout d'abord sur une incompatibilité des programmes et des règlements. Les modes de financement des projets communs sont restés longtemps largement différenciés avec, de 2004 à 2007, des acteurs russes et biélorusses soutenus par le programme TACIS et des acteurs baltes inscrivant leur participation dans le cadre des fonds structurels européens. Ces deux sources de financement suivaient des réglementations et des temporalités de décaissement des crédits qui n'étaient pas harmonisées. En 2007, une simplification est décidée par les autorités européennes : les projets transfrontaliers et transnationaux sont dès lors financés par le FEDER couplé à l'instrument européen de voisinage et de partenariat (IEVP) et aux budgets nationaux. Cette évolution apparaît relativement propice à une coopération accrue avec la Biélorussie mais ne surmonte pas les blocages concernant un rapprochement avec la Russie qui ne fait pas partie du cadre géographique de la politique européenne de voisinage. Au-delà de ces questions de compatibilité, les budgets de l'IEVP apparaissent relativement faibles et sont concentrés sur des zones stratégiques comme la région frontalière Pologne-Biélorussie-Ukraine ou les alentours de Kaliningrad (Lituanie-Pologne-Russie). La coopération 
transfrontalière ne constitue pas une priorité des budgets nationaux. Du côté de l'Union européenne, la plupart des instruments de la politique régionale révèlent également une faible attention portée aux interactions entre le territoire communautaire et les partenaires externes. La période 2007-2013 a été enfin marquée par des retards de signature de la Russie engendrant une entrée tardive des acteurs russes dans les appels à projets.

Des barrières mentales. Les géographes Vladimir Kolossov, Maria Zotova et Alexander Sebentsov ont réalisé un sondage parmi 675 étudiants lituaniens, polonais et russes des universités de Gdańsk, Klaipèda et Kaliningrad sur leur perception des rapports entre les deux pays de l'UE et l'Oblast de Kaliningrad ${ }^{52}$. Les auteurs montrent que si $61,1 \%$ des répondants russes ont déjà visité la Pologne et 47,6\% la Lituanie, seulement $12 \%$ des étudiants de Gdańsk et 29,6\% des étudiants de Klaipèda se sont déjà rendus à Kaliningrad. Les étudiants polonais ont une vision relativement négative de Kaliningrad qu'ils associent à un ensemble de mots dépréciatifs : point sur la carte, région sous-développée, vodka et ivresse, froid, SIDA, Staline et Poutine, mafia, camps de concentration, frontière difficile. Seule la mention d'une essence bon marché semble apporter un angle plus positif. Pourtant, côté russe, $69,8 \%$ des répondants voient les perspectives de la région dans une coopération avec l'UE et seulement $11 \%$ avec les autres régions de la Russie. Afin de mettre en avant une persistance des stéréotypes et l'absence d'interconnaissance, les auteurs parlent de renforcement de la frontière (rebordering).

\subsection{LA BALTIQUE ORIENTALE : UNE DIVERSIFICATION PROGRESSIVE DES PARTENARIATS VERS LES ÉTATS DE LA CEI}

Face aux nombreuses contraintes et dépendances qui affectent les villes et ports baltiques dans leur relation avec la Russie et dans un contexte au cours de l'année 2014 d'escalade des sanctions côté européen, une diversification des partenariats notamment vers les États de la CEI est apparue indispensable.

52. Résultats présentés lors du sixième Festival international de géopolitique de Grenoble (2-6 avril 2014), voir la vidéo de la conférence sur le site du festival : http://tv.grenoble-em.com (consulté le 10/08/2014). 
Au niveau portuaire, les liaisons est-ouest s'appuient principalement sur une complémentarité entre transport maritime et transport ferroviaire. Le rail représente $50 \%$ du transport du fret dans les États baltes. Leur hinterland est relativement différencié si l'on excepte la place importante de la Russie. La Lituanie accueille des flux provenant de la plupart des États post-soviétiques avec d'importants échanges tissés avec la Biélorussie. Le port de Klaipèda est ainsi le principal port d'exportation des engrais biélorusses vers les USA, l'Union européenne et même la Chine. Début 2013, l'entreprise biélorusse OAO Belaruskali a d'ailleurs acheté $30 \%$ du terminal vraquier de Smelte pour 30 millions de dollars, ce qui en fait le premier investisseur étranger dans le port de Klaipèda. La Lettonie est caractérisée par une plus grande concentration des partenaires commerciaux. La Biélorussie, l'Ukraine, la Moldavie, le Kazakhstan ou l'Ouzbékistan utilisent les ports lettons, ce qui n'est pas le cas des pays du Caucase. L'Estonie semble se situer, quant à elle, dans une position intermédiaire avec une grande variété de partenaires sans pour autant que certains d'entre eux se distinguent.

Au niveau des municipalités, il semble que la plupart des autorités locales ont profité d'un héritage culturel commun et de contacts anciens pour maintenir des échanges avec des villes de la CEI. L'exemple des visites à l'étranger des départements et de l'administration centrale de la municipalité de Riga, notamment dans le cadre de réseaux institutionnels et de projets européens, le montre. Les capitales baltes et les villes du golfe de Finlande concentrent bien entendu une grande partie des voyages de délégations. En dehors de l'espace baltique, les visites à l'étranger se dirigent pourtant à la fois vers des métropoles européennes, comme Bruxelles ou Berlin, et vers des villes de l'ancien bloc soviétique, comme Minsk ou Kiev, avec une place particulière pour Moscou. La nature des contacts établis par Riga à l'étranger met en évidence une situation intermédiaire entre une insertion européenne progressive et le maintien d'échanges avec des partenaires historiques. La cartographie des villes les plus visitées par les acteurs municipaux de Riga dessine d'ailleurs une écharpe de Bruxelles à Moscou. La position géographique de la municipalité lui permet de nourrir une double ambition comme possible future métropole européenne et comme éventuel pont vers l'est (Escach, 2014). 


\subsection{LA BALTiQue orientale :}

\section{LA CONSTRUCTION PROGRESSIVE DE CORRIDORS EURASIATIQUES}

Alors que les pays européens ont connu une crise économique sans précédent en 2008-2009, certains pays émergents, principalement asiatiques, continuent d'afficher des croissances exceptionnelles. Selon le FMI, la Chine est la première puissance mondiale depuis 2014. Le discours de Bruges du président chinois Xi Jinping du $1^{\text {er }}$ avril 2014 sur les relations entre l'Union européenne et la Chine rappelle l'importance de construire des ponts de croissance entre l'Asie et l'Union européenne.

L'espace eurasiatique constitue depuis le milieu de la décennie 2000 un hinterland de quelques ports baltiques comme en témoigne la multiplication des trains-blocs depuis la façade orientale de la mer Baltique. Un train-bloc - ou block train - est un train complet de marchandises acheminées directement du point de départ au point de destination sans remaniement intermédiaire. La réalisation la plus aboutie est le train shuttle Viking, lancé en 2003, reliant Klaipèda (Lituanie) à Odessa (Ukraine), qui transporte environ 40000 EVP par an. D'autres trains-blocs sont également en service entre les ports de la région et la CEI. Le Baltica transit relie Riga et Almaty (Kazakhstan), avec deux trains de conteneurs par semaine et un temps de transit de 8 à 10 jours. Le Moscou express est un train de conteneurs régulier, opérant depuis 2007, de Tallinn à Moscou. Depuis janvier 2013, un train relie le terminal conteneur de Muuga (port de Tallinn) à Kalouga trois fois par semaine. Ce dernier exemple illustre un nouveau développement des trains-blocs dans la région, en étroite relation avec l'activité industrielle, notamment l'usine PSA de Kalouga. En effet, ce dernier vise avant tout à assurer l'approvisionnement en pièces détachées des usines automobiles de Kalouga. Les projets contemporains des opérateurs de trains-blocs renforcent cette ouverture eurasiatique puisqu'un trainbloc entre Shanghai et Kalouga est en cours de développement, proposant une alternative à la voie maritime entre Asie et Europe occidentale. D'autres projets ferroviaires sont envisagés dans la région, comme ceux d'AS Baltic Rail, société de transport ferroviaire basée en Estonie et en Pologne, qui cherche à développer des services réguliers de trains de conteneurs à partir de Šeštokai (Lituanie) vers Saint-Pétersbourg, Moscou et l'Asie centrale. Le choix de la gare de Šeštokai n'est pas anodin car il s'agit du terminus oriental des lignes ferroviaires à gaba- 
rit européen. L'idée est de transformer ce handicap en atout pour le développement de l'interopérabilité des réseaux. Par ailleurs, les ports cherchent, via leur communication, à attirer les partenaires asiatiques, qu'il s'agisse des États enclavés d'Asie centrale ou de la Chine : sites Internet, revues trilingues rédigées en anglais, russe et chinois...

Du côté des municipalités, les liens avec l'Asie y compris lointaine, sont également l'objet de stratégies relativement élaborées. L'aéroport d'Helsinki et la compagnie aérienne Finnair ont multiplié depuis l'ouverture de la liaison avec Shanghai en 2003 les vols vers les métropoles asiatiques en utilisant notamment la circonférence terrestre. Parallèlement, les réseaux et projets baltiques associant des acteurs municipaux intègrent la croissance asiatique, comme en témoigne le projet européen « BaltMet Promo » (2009-2011) mené par des métropoles baltiques dont Riga. Dans le cadre du projet, des enquêtes ont été conduites par la compagnie Foresight Marketing et l'institut de recherche intégrée de Tokyo auprès d'un panel d'habitants de la ville susceptibles de se rendre dans les pays baltes. Il s'agissait de définir des produits touristiques facilement exportables en Asie. Trois espaces lisibles pour les touristes de la capitale nippone ont été identifiés : les capitales baltes, les capitales scandinaves et le binôme Berlin/Varsovie. L'ensemble balte aurait intérêt à développer des produits touristiques autour de trois dimensions : une envie de nature, un univers médiéval et une ambiance de contes et légendes. À côté du volet « tourisme», le projet a permis le développement d'un volet « investissement » avec le financement d'un guide de présentation des villes à destination des investisseurs asiatiques et d'un volet " cinéma » autour d'échanges culturels entre cinéastes.

\section{Conclusion}

L'espace baltique, en raison de sa situation géographique aux confins de l'Europe et de la Fédération de Russie, s'affirme comme un objet d'étude central pour l'appréhension des relations Est-Ouest. La Baltique orientale constitue un laboratoire de ces raccordements qui s'effectuent à plusieurs échelles. Les relations entre acteurs locaux baltiques et russes, bien que marquées par certains obstacles, ne subissent pas nécessairement les blocages étatiques géopolitiques car ces acteurs agissent dans le cadre d'interactions plus flexibles et plus fonction- 
nelles. Les municipalités européennes disposent de peu d'outils institutionnels pour une telle coopération, la Russie étant le seul pays riverain de la mer Baltique à ne pas être membre de l'Union européenne tout en ne bénéficiant pas des cadres, certes limités, fixés par le Partenariat oriental. Les ports, quant à eux, doivent engager une diversification des partenariats afin de réduire leur dépendance envers les transits en provenance de Russie. Dans un tel contexte, les échanges avec les États de la CEI pour la plupart également membres du Partenariat oriental et, plus largement, avec l'Asie centrale et orientale se multiplient, réactivant des contacts, des infrastructures et des dispositifs spatiaux qui semblaient dépassés. Ces réseaux hérités, physiques ou informels, répondent en réalité géographiquement tout aussi bien aux dynamiques les plus contemporaines qu'un ancrage occidental unique et exclusif. La construction d'un espace transnational baltique s'inscrit dans une volonté politique de créer des relais là où l'éloignement des centres européens aurait pu ne laisser que des marges. Les interactions, continentales voire intercontinentales, que les réseaux baltiques suscitent, confirment la vocation de la région à devenir un gateway. La Northern Range (pour les ports) ou la Dorsale européenne (pour les métropoles) apparaissent comme des espaces structurants que les acteurs baltiques souhaitent relier aux principaux pôles de croissance orientaux. En ce sens, l'interterritorialité ne peut bénéficier à l'espace baltique que parce qu'elle se définit au sens large. Le rapprochement entre les différentes rives, par de multiples coopérations, sert surtout à structurer et à organiser passages et mises en relation, au sein d'un véritable espace médian. Les ports et municipalités baltes trouvent à l'est de l'Union européenne, notamment sur l'axe mer Baltique-mer Noire, de nouvelles perspectives de développement. Ils apparaissent, dans cette configuration, comme susceptibles de peser fortement sur la diplomatie régionale et d'insuffler des dynamiques comme l'avaient fait les États nordiques pour eux au moment de la transition démocratique ${ }^{53}$.

Ces propriétés de l'espace baltique, encore émergentes, sont longtemps restées impensées dans l'appréhension de la métropolisation et de l'européanisation car elles ne peuvent être révélées par l'étude d'ac-

53. Voir à ce sujet la thèse de Katerina Kesa soutenue le 7 mai 2015 à l'INALCO sous la direction de Charles Urjewicz et intitulée Pays récepteurs d'assistance étrangère et pays donneurs : la place et le rôle des Etats baltes entre pays nordiques et États postsoviétiques au prisme de l'action de parrainage (1985-2013). 
teurs majeurs (UE, États et firmes transnationales) ou la prise en compte d'indicateurs économiques ou politiques majeurs (flux d'IDE, sièges sociaux, principaux hubs conteneurs, réseaux de lobbying à Bruxelles). Le choix d'une entrée par les municipalités et les ports donne une visibilité à une somme de coopérations territoriales dont les actions ne sont pas quantifiables économiquement et sont rarement considérées comme stratégiques. L'addition de ces interactions « à bas bruit » finit tout de même par révéler une géographie structurante de l'Europe et de ses marges. La mer Baltique est composée, pour une part non négligeable, de liens faibles c'est-à-dire de relations volontaristes, médiées et discontinues, en recomposition permanente, qui ne deviennent structurantes que par des effets d'accumulation. Les échanges portuaires ou les réseaux de municipalités mettent en relief des zones grises de la carte, des impensés géographiques car ils révèlent une insertion européenne et mondiale différente, par des processus ténus, discrets et souples mais tout aussi stratégiques.

\section{BIBLIOGRAPHIE}

Bayou Céline \& Chillaud Matthieu, dir. (2012), Les États baltes en transition. Le retour à l'Europe, Bruxelles, Bern, Berlin, Francfort-sur-leMain, New York, Oxford, Vienne : Peter Lang International Academic Publishers, $264 \mathrm{p}$.

BLANC-NoËL Nathalie (2002), La Baltique: une nouvelle région en Europe, Paris : L'Harmattan, $174 \mathrm{p}$.

BRownING Christopher (2005), «Introduction », Remaking Europe in the Margins. Northern Europe after the Enlargements, Aldershot: Ashgate Pub Co, pp. 1-10.

BRUnet Roger (1992), Les mots de la géographie, Paris/Montpellier : Reclus-La Documentation française, $470 \mathrm{p}$.

Champonnois Suzanne (2002), « La Hanse dans la Baltique », Le Monde de Clio ; http://www.clio.fr.

DAutencourt Vincent (2011), « La frontière Estonie-Russie entre absence de traité juridique et réalités quotidiennes », Études Finno-ougriennes, tome 42, pp. 159-196. 
Debrié Jean, Eliot Emmanuel \& Soppé Martin (2005), « Un modèle transcalaire des nodalités et polarités portuaires. Exemple d'application au port de Hambourg », Mappemonde, vol. 3, n 79 ; http://mappemonde. mgm.fr.

Dellenbrant Jan Áke (1999) «The Baltic Sea Co-opération, Visions and Realities, » Nordic Region-Building in a European Perspective, Aldershot: Ashgate, pp. 83-97.

Dollinger Philippe (1964), La Hanse (XII - -XVII siècles), Paris : Aubier, $559 \mathrm{p}$.

EsCACH Nicolas (2014), Réseaux de villes et recompositions interterritoriales dans l'espace baltique, Thèse de doctorat soutenue à l'ENS de Lyon le 14 novembre 2014 sous la direction de Lydia Coudroy de Lille et Boris Grésillon, 456 p.

EsCACH Nicolas \& SerRy Arnaud (2014), « Les méditerranées ou l'émergence de régionalisations réticulaires », in Nicolas Escach, Géographie des mers et des océans : Capes-Agrégation histoire-géograhie, Paris : Dunod, $448 \mathrm{p}$.

Escach Nicolas \& Serry Arnaud (2013), « Les ports de la mer Baltique entre mondialisation des échanges et régionalisation réticulaire », Géoconfluences; http://geoconfluences.ens-lyon.fr.

FRÉMONT Armand (2011), La Normandie en mal de métropole ?, Rapport rédigé au nom de la commission intergroupe de réflexion et de proposition sur la réunification de la Normandie ; http://normandie-metropole. fr, $9 \mathrm{p}$.

Halbert Ludovic, Cicille Patricia, Pumain Denise \& Rozenblat Céline (2012), Quelles métropoles en Europe ?, analyse comparée, Paris : Documentation française, $20 \mathrm{p}$.

HAMEdinger Alexander \& WolfFhardt Alexander (2011), The Europeanisation of Cities, Policies, Urban Change and Urban Networks, Amsterdam: Techne Press, 248 p.

Helmryd Cécile (1993), La Baltique : regards nordiques sur une région en devenir, Mémoire de fin d'études sous la direction de Jean-Christophe Romer, Université de Paris I, 114 p.

Joenniemi Pertti (1991), "Co-operation in the Baltic Sea Region: Needs and prospects," Research Report, $\mathrm{n}^{\circ}$ 42, Tampere Peace Research Institute (TAPRI), $165 \mathrm{p}$.

Joenniemi Pertti \& Wæver Ole (1992), "Regionalization around the Baltic Rim: Notions on Baltic Sea Politics," Nordic Seminar and Workinggroup reports, The Nordic Council, $\mathrm{n}^{\circ} 521,38 \mathrm{p}$. 
Jervell Sverre, Kukx Mare \& Joenniemi Pertti, dir. (1992), The Baltic Sea area, a region in the making, Oslo: Europa-Programme in cooperation with the Baltic Institute, $241 \mathrm{p}$.

KERN Kristine \& LÖFFELSEND Tina (2004), "Governance beyond the nationstate : transnationalization and europeanization of the Baltic Sea Region," Discussion Papers, Abteilung Zivilgesellschaft und transnationale Netzwerke des Forschungsschwerpunkts Zivilgesellschaft, Konflikte und Demokratie des Wissenschaftszentrums Berlin für Sozialforschung, $\mathrm{n}^{\circ} 4,35 \mathrm{p}$.

Le Bourhis Éric (2009), « Le port pétrolier de Ventspils : une parabole des relations russo-lettones », Regard sur l'Est, dossier n ${ }^{\circ} 52$; www. regard-est.com.

Mertelsmann Olaf \& Pirrimäe Kaarel (2012), The Baltic Sea Region and the Cold War, Francfort-sur-le-Main : Peter Lang International Academic Publishers, $291 \mathrm{p}$.

Neumann Iver Brynild (1998), Uses of the Other: the 'East'in European identity formation, Minneapolis: University of Minnesota Press, $304 \mathrm{p}$.

OCDE (2009), «Finlande», Examens environnementaux de l'OCDE, Paris : Éditions OCDE, 292 p.

PARKer Noel (2000), "Integrated Europe and its Margins. Action and Reaction," in Noel Parker, Margins in European Integration, Houndmills, Basingstoke: St. Martin's Press, pp.3-27.

Perchoc Philippe (2006), « La ligue hanséatique », Nouvelle Europe, 11 novembre ; http://www.nouvelle-europe.eu.

Perko Susanna (1996), Nordic-Baltic Region in Transition, Tampere: Tampere Peace Research Institute, $170 \mathrm{p}$.

RichARD Yann (2011), « Pays Baltes-Russie. L'impossible coopération tranfrontalière ? », L'Espace politique, vol. 14, n 2 ; http://espacepolitique.revues.org.

SERRY Arnaud (2013), « Circulations commerciales à la frontière entre Russie et Union européenne en Baltique orientale », in Nicolas Lebrun \& Nathalie Lemarchand, Commerce et Discontinuités, Arras : Artois Presses Université, pp. 79-90.

SERRY Arnaud (2005), « Les ports de la Baltique orientale : persistance des territoires et mutations », Le courrier des pays de l'Est, $\mathrm{n}^{\circ} 1048$ : «L'espace baltique, isthme russo-européen ? », pp. 40-50. 
Thorez Pierre (2011), " Les enjeux portuaires de la Russie en mer Baltique », Territoire en mouvement, revue de géographie et aménagement, $\mathrm{n}^{\circ} 10 ;$ http://tem.revues.org.

VergnAud Guillaume (2011), À la recherche de la maille territoriale intermédiaire : approche comparée des recompositions et de l'aménagement des territoires en Rhône-Alpes (France) et en Catalogne (Espagne), Thèse de doctorat de l'ENS de Lyon, 327 p. 